\title{
Quadrivalvular non-bacterial thrombotic endocarditis in a patient with clear cell cervical cancer
}

\author{
Valentin Gabelmann ${ }^{1}$, Felix Grabs ${ }^{1}$, Simon Diestelmeier ${ }^{1}$, Felix Heindl ${ }^{2}$, Markus \\ Vosseler $^{1}$, Thomas Münzel ${ }^{1}$, Ingo Sagoschen ${ }^{1}$, and Johannes Wild ${ }^{1}$ \\ ${ }^{1}$ Johannes Gutenberg University Mainz \\ ${ }^{2}$ Erlangen University Hospital
}

January 5, 2022

\begin{abstract}
Non-bacterial thrombotic endocarditis (NBTE) is a rare finding, which is mostly associated with malignant diseases leading to hypercoagulability. We report the case of a severe quadruple valve non-bacterial thrombotic endocarditis in a patient with clear cell cervical cancer.
\end{abstract}

\section{Introduction}

Whereas venous thromboembolism (VTE) is a frequent clinical event in patients with cervical cancer [1], nonbacterial thrombotic endocarditis (NBTE), formerly known as marantic endocarditis, has rarely been described associated to gynecological malignancy [2] and in most of the cases, the diagnosis was confirmed postmortem [3, 4]. NBTE is considered as a manifestation of an overall prothrombotic state [5] characterized by valve associated masses consisting of platelets and fibrin in absence of bacteria and without inflammatory destruction of the heart valves.

We report a case of NBTE of all heart valves and the atrial septum in a patient suffering from clear cell cervical cancer resulting in fatal venous and arterial thromboembolic events.

\section{Case Report}

A 52-year-old female patient was referred to our university medical center with suspected acute infectious endocarditis (IE). Nine days before, an ischemic stroke of the left middle cerebral artery had been diagnosed at the referring hospital. ECG showed no evidence of atrial fibrillation supporting cardioembolic events. A transesophageal echocardiography was performed and vegetations adherent to the in-situ port catheter and the aortic valve were found. An acute infective endocarditis was suspected and calculated antibacterial treatment with ampicillin and gentamicin was initiated. Because of respiratory distress a computed tomography of the pulmonary arteries was performed, which revealed bilateral pulmonary embolism. Thereupon, the patient was referred to our tertiary care hospital for further therapy.

Medical history revealed that the patient had clear cell carcinoma of the cervix at an advanced stage (FIGO IV4A). The carcinoma had been diagnosed one year ahead of the current admission and initially treated with lymphadenectomy, and concurrent platinum-containing radiochemotherapy. With recently identified lymphogenic progression, therapy with Navelbine had been initiated. In addition, the patient had a history of pulmonary embolism in November 2020 since then followed by oral treatment with the direct factor Xa inhibitor Edoxaban for therapeutic anticoagulation.

On admission to our unit, the patient presented with severe hemodynamic instability reflected by tachycardia and hypotension. Respiratory distress required a high flow oxygen support (10 l/min) via facial mask. 
The patient had no fever. Laboratory findings showed moderately elevated c-reactive protein (137mg/l), normal procalcitonin $(0.5 \mathrm{ng} / \mathrm{ml})$ an normal leukocyte counts $(9.43 / \mathrm{nl})$. Moreover, we detected mild thrombocytopenia $(115 / \mathrm{nl})$, anemia (serum hemoglobin $7,3 \mathrm{~g} / \mathrm{dl}$ ) and highly elevated d-dimers $(15 \mathrm{mg} / \mathrm{l})$. Notably, Troponin I $(11462 \mathrm{pg} / \mathrm{ml})$ and BNP $(2624 \mathrm{pg} / \mathrm{ml})$ were as well elevated (Tab. 1 for relevant laboratory findings on admission).

Bedside transthoracic echocardiography on admission suggested large vegetations affecting the aortic valve, so we performed an urgent transesophageal echocardiography. Consistent with the earlier examination, we found large vegetations, but not only on the aortic valve (Fig. 1 ) also affecting the mitral (Fig. 2 A/B ) and the tricuspid valve (Fig. 2C ) with large floating masses within the right ventricle extending over the pulmonary valve into the pulmonary artery (Fig. 2D ). Notably, although the vegetations were very pronounced, there was no destruction of the valves and only moderate insufficiencies could be visualized (Fig. 1B/2B/2C ). We also detected vegetations adherent to the right side of the atrial septum (Fig. 3 ) and impaired systolic function of the left ventricle.

We initiated microbiological diagnostics by taking three pairs of blood cultures as well as next generation sequencing (NGS)-based diagnostics for pathogen identification and extended the antibiotic therapy by Flucloxacillin according to guidelines for IE. Given the malignant underlying disease and the low values of PCT, we additionally started therapeutic anticoagulation with unfractionated heparin and performed extended thrombophilia and coagulation diagnostics (Tab. 2 ).

After multidisciplinary discussion of the case, a joint decision did not see surgical therapy as a favorable option. Following circulatory deterioration the patient died on our ICU due to shock-associated multiple organ failure only two days later.

\section{Discussion}

Nonbacterial thrombotic endocarditis (NBTE) is a rare clinical finding and is likely underdiagnosed, although it is a serious manifestation of cancer-related hypercoagulability and a potentially life-threatening source of thromboembolism [5]. NBTE was first described by Ziegler in 1888 as fibrinous efflorescence on heart valves [6]. In 1936, the term was renamed "nonbacterial thrombotic endocarditis" by Gross and Friedberg [7] and defined as deposition of fibrin and platelets on heart valves without evidence of microorganisms. Most cases of NBTE are detected post mortem, and autopsy reports indicate an incidence of $1.2 \%$ [8]. Anatomically, the aortic valve is most commonly affected, followed by the mitral valve. Pulmonary and tricuspid valves are rarely affected [8]. Since NBTE is a rare finding, multivalvular NBTE is even rarer and quadrivaluvular NBTE is a real rarity with very few published cases [9-11].

The pre-mortem diagnosis of NBTE is usually made on the basis of clinical and echocardiographic findings in conjunction with exclusion of an infectious cause of endocarditis. Because an autopsy (required by german law) was refused by the family, our diagnosis was also made based on echocardiographic imaging after interdisciplinary discussion and after negative results of microbiologic diagnostics. All of our conventional blood cultures and blood culture-negative endocarditis (BCNE)-diagnostics remained sterile. In addition, we used commercially available next generation sequencing (NGS)-based diagnostics for pathogen identification (Noscendo DISQVER\&) that can detect bacteria, DNA viruses, fungi, and parasites in a single assay [12]. This highly sensitive assay also showed no evidence of a bacterial or fungal pathogen, so we are confident in diagnosing NBTE in our patient.

Regarding differential diagnoses, cardiac metastasis is also a very rare disease [13] and involvement of heart valves is an uncommon site for manifestation [14]. Evidence of cervical cancer metastasizing to the heart is available from several case reports in recent decades, but none involved the heart valves which makes the diagnosis very unlikely.

In a prospective study, NBTE was significantly more common in cancer patients [15]. Among gynecologic malignancies, ovarian cancer is the most common cancer associated with NBTE [16]. To our knowledge, neither a case of NBTE in a patient with clear cell cervical cancer nor a case of quadrivalvular NBTE in any 
gynecologic malignancy has been published so far.

Disseminated intravascular coagulation (DIC) can be detected in most NBTE patients [5], indicating a poor prognosis overall. We would like to emphasize that in our case, NBTE developed in spite of preexisting anticoagulation. Our patient did not meet the criteria for DIC based on the Overt DIC-score by the International Society for Thrombosis and Hemostasis [17] consisting of low platelet count, elevated levels of a fibrin-related marker, prolonged prothrombin time and decreased fibrinogen levels. Nevertheless, we found high levels of D-Dimers and thrombin-antithrombin complexes (TAT). The presence of TAT indicates ongoing, intravascular thrombin formation as well as the consumption of antithrombin and is associated with DIC [18].

In addition, the patient showed severe venous and arterial thromboses. Thus, on the one hand, the stroke was likely caused by arterial thrombosis resulting from dislocated thrombotic material from the NBTE of the aortic valve. The occurrence of stroke is a disastrous prognostic sign in NBTE patients with a 6-month lethality of $80 \%$ [19]. On the other hand, the acute pulmonary embolisms can well be attributed to carryover from the marked thrombotic masses in the area of the tricuspid and pulmonary valves.

\section{Conclusion}

To our best knowledge, we report the first case of a quadrivalvular non-bacterial endocarditis in a patient with a clear cell cervical cancer.

\section{Author's Contribution}

VG: Imaging, drafting of the article; FG: Taking the medical history of the patient; SD: Advice on imaging; FH/MV/TM/IS: critical revision of article; JW: Imaging, concept and drafting of the article.

\section{Conflict of interest}

The authors report no conflict of interests.

\section{References}

1. Bleker, S.M., et al., Clinical course of upper extremity deep vein thrombosis in patients with or without cancer: a systematic review. Thrombosis Research, 2016. 140 : p. S81-S88.

2. Orfanelli, T., et al., Nonbacterial thrombotic endocarditis: A rare manifestation of gynecologic cancer. Gynecol Oncol Rep, 2016.17 : p. 72-4.

3. Erturk, N.K., et al., Synchronous Ovarian and Endometrial Endometrioid Adenocarcinoma Presenting with Nonbacterial Thrombotic Endocarditis and Pulmonary Thromboembolism: Adenocarcinoma with Thrombotic Events. Case Rep Obstet Gynecol, 2015. 2015 : p. 825404.

4. Aryana, A., D.J. Esterbrooks, and P.C. Morris, Nonbacterial thrombotic endocarditis with recurrent embolic events as manifestation of ovarian neoplasm. J Gen Intern Med, 2006. 21 (12): p. C12-5.

5. el-Shami, K., E. Griffiths, and M. Streiff, Nonbacterial thrombotic endocarditis in cancer patients: pathogenesis, diagnosis, and treatment. Oncologist, 2007. 12 (5): p. 518-23.

6. Ziegler, E., Ueber den Bau und die Entstehung der endocaridtis chen Efflorescenzen. Ver Kong Inn Med, 1888. 7 : p. 339-343.

7. Gross, L. and C.K. FRIEDBERG, Nonbacterial thrombotic endocarditis: classification and general description. Archives of internal medicine, 1936. 58 (4): p. 620-640.

8. Lopez, J.A., et al., Nonbacterial thrombotic endocarditis: a review. Am Heart J, 1987. 113 (3): p. 773-84.

9. Durie, N.M., et al., Quadrivalvular marantic endocarditis (ME) mimicking acute bacterial endocarditis $(A B E)$. Heart Lung, 2007.36 (2): p. 154-8.

10. Vlismas, P.P., et al., Quadravalvular Noninfectious Endocarditis. JACC Case Rep, 2019. 1 (3): p. 350-354. 
11. Laco, J., I. Steiner, and E. Havel, Nonbacterial thrombotic endocarditis involving all four cardiac valves. Pathol Res Pract, 2008.204 (10): p. 757-61.

12. Kattner, S., et al., Next-Generation Sequencing-Based Decision Support for Intensivists in Difficultto-Diagnose Disease States: A Case Report of Invasive Cerebral Aspergillosis. A A Pract, 2021.15 (5): p. e01447.

13. Hoppe, U.C., et al., [Heart tumors-their manifestation through uncharacteristic symptoms]. Dtsch Med Wochenschr, 1997.122 (17): p. 551-7.

14. Butany, J., et al., A 30-year analysis of cardiac neoplasms at autopsy. Can J Cardiol, 2005. 21 (8): p. $675-80$.

15. Edoute, Y., et al., Cardiac valvular vegetations in cancer patients: a prospective echocardiographic study of 200 patients. Am J Med, 1997. 102 (3): p. 252-8.

16. Delgado, G. and J.P. Smith, Gynecological malignancy associated with nonbacterial thrombotic endocarditis (NBTE). Gynecol Oncol, 1975. 3 (3): p. 205-9.

17. Gando, S., et al., A multicenter, prospective validation of disseminated intravascular coagulation diagnostic criteria for critically ill patients: comparing current criteria. Crit Care Med, 2006. 34 (3): p. 625-31.

18. Mei, H., et al., Evaluation the combined diagnostic value of TAT, PIC, tPAIC, and sTM in disseminated intravascular coagulation: A multi-center prospective observational study. Thromb Res, 2019.173 : p. 20-26.

19. Yoo, J., et al., Outcome of Stroke Patients with Cancer and Nonbacterial Thrombotic Endocarditis. J Stroke, 2020. 22 (2): p. 245-253.

\section{Tables}

\section{Table 1}

Laboratory findings on admission. eGFR $=$ estimated glomerular filtration rate, LDH = lactatedehydrogenase, $\mathrm{CK}=$ Creatine kinase, $\mathrm{BNP}=$ Brain natriuretic peptide, $\mathrm{CRP}=$ C-reactive protein.

\section{Table 2}

Extended thrombophilia and coagulation diagnostics. INR $=$ International normalized ratio, APTT $=$ Activated Partial Thromboplastin Time.

\section{Figures and Figure Legends}

\section{Figure 1}

(A) Transesophageal echocardiography showing a large masse adhering to the aortic and pulmonary valves as well as the atrial septum. AV = aortic valve, $\mathrm{PV}=$ Pulmonary valve, AS = atrial septum.(B) Color doppler echocardiography demonstrating aortic valve insufficiency. (C) 3-D trans-esophageal echocardiography of the aortic valve. Arrows highlighting the vegetations.

\section{Figure 2}

(A) 2-D Transesophageal echocardiography and (B) color doppler echocardiography with vegetations on the mitral valve (MV) and the resulting insufficiency. (C) Imaging of the tricuspid valve (TV) affected by NBTE and moderate to severe insufficiency. (D)3-D and 2D-transesophageal echocardiography of the ventricular outflow tract (RVOT) and the pulmonary valve (PV). Arrows highlighting the vegetations.

\section{Figure 3}

2-D Transesophageal echocardiography and X-plane images of the atrial septum (AS), the left atrium (LA) and the right atrium (RA) with arrows highlighting the vegetations.

\section{Hosted file}


Figures Case 2.pptx available at https://authorea.com/users/454076/articles/551814quadrivalvular-non-bacterial-thrombotic-endocarditis-in-a-patient-with-clear-cellcervical-cancer 